\author{
ANNALES \\ POLONICI MATHEMATICI \\ XXXI (1076)
}

\title{
On $D$-connected sets in the space $V^{q}$
}

\author{
by S. Topa (Krraków)
}

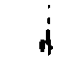

Abstract. Let be given an arbitrary topological space $X$ and its arbitrary de. composition $D:=\left\{X_{\lambda,\}_{\lambda \in A}}\right.$ into disjoint sets. Wo take $\Lambda_{\mathfrak{v}} \subset \Lambda$ arbitrarily and by $\Lambda_{0}^{*}$ wo denote the family of all $\lambda^{*} \epsilon \Lambda_{0}$ such that for erery $\lambda \neq \lambda^{*}, \lambda \in \Lambda_{0}$, we have $\operatorname{cl}\left(X_{\lambda^{*}}\right) \cap X_{\lambda}=\emptyset$ (cl being the operation of closuro) and wo put

$$
A:=\bigcup_{\lambda \in A_{0}} X_{\lambda} \text { and } A^{*}:=\bigcup_{\lambda \in A_{0}^{*}} X_{\lambda} .
$$

Any function $f: A \rightarrow R$ being constant on evory $X_{\lambda} \subset A$ is called $D$-function on $A$.

Derinition. The set $A$ is said to be $D$-connected in $X$ iff (1) $A^{*} \neq \varnothing$. (2) For every $\lambda \in \Lambda_{0}$ wo have $\operatorname{cl}\left(X_{\lambda}\right) \cap A^{*} \neq \varnothing$. (3) For every $D$-function $f$ on $A$ the condition

$$
f\left(\operatorname{cl}\left(X_{\lambda}\right) \cap A^{*}\right) \subset \operatorname{cl}\left(f\left(X_{\lambda}\right)\right) \quad \text { for all } \lambda \in \Lambda_{0}
$$

implies the constancy of $f$ on $A$.

In the paper a certain method of construction of $D$-connected sets in $X:=\nabla^{Q}$ for the decomposition $D$ consisting of all $G L\left(V^{\prime}\right)$-orbits in $T^{q}$ is presented, GL $(\nabla)$ denoting the general linear group of a given topological vector spaco $V$ and $q$ an arbitinry natural number.

I. Introduction. In this paper we give a method for the determination of some families of $D$-connected sets in $V^{a}$ for $D$ being the decomposition of $\nabla^{q}$ into orbits of the lineal group $\mathrm{GL}(V)$ acting on $V^{q}$ in the natural way (called $\mathrm{GL}(V)$-decomposition), $\nabla$ is a given topological vector space and $q$ a natural number (see Theorems 1 and 2). The problem of the determination of all $D$-connected sets in $\nabla^{a}$ is open.

The notion of the $D$-connected set (see Definition 6 ) is original. To give the definition let us suppose we are given a topological space $X$ and an arbitrary decomposition $D$ of $X$ into disjoint sets. For example, $D$ may be considered as the decomposition of $X$ into orbits of an abstract group $G$ acting on $X$ arbitrarily (such decomposition $D$ being called G-decomposition).

If $A$ is a subspace of $X$, then we denote by $D_{A}$ the decomposition of $A$ induced by $D$.

Definition 1. We say that a set $A \subset X$ reaches a set $B \subset X$ iff $B \cap c l A \neq \emptyset$ (cl being the operation of closure). 
Definizion 2. Te say that a set $A \subset X$ is concentrating on a subset $B$ of $A$ iff each element of $D_{A}$ reaches the set $B$.

Defrinition 3. A function $f: A \rightarrow R, A \subset \bar{X}$, is said to be $D$-continuous iff for each subset $C$ of each element of $D_{A}$ there is $f(\operatorname{cl} C) \subset \operatorname{cl}(f(C))$.

Disfinition 4. A function $f: A \rightarrow R$ is called $D$-function iff it is constant on each element of $D_{A}$.

Defrnirion 5. A set $A \subset X$ is called $D$-admissible iff it is the union of a family of elements of $D$.

For $A \subset X$ we denote by $A^{*}$ the union of all those elements of $D_{A}$ which do not reach any other elements of $D_{A}$.

Now the basic

Definimion 6 . Let $A$ bo a given $D$-admissible set in $X$. Then $A$ is called a $D$-connected set in $X$ iff (1) $A^{*} \neq \emptyset,(2) A$ is concentrating on $A^{*}$, (3) for each $D$-function $f: A \rightarrow R, D$-continuous on $A^{*}$, we obtain that $f$ is constant.

A theory of $D$-comnected sets has not yet been elaborated. We may only give a result concerning some sufficient conditions for a given family of $D$-connected sets, the union of which is also a $D$-conuected set (see Proposition 1).

It may be observed that any element of $D$ is a $D$-connected set in $X$. Such $D$-connected sets are called trivial.

In the following we are only interested in non-trivial $D$-conmected sets. Of course, not every decomposition $D$ of $X$ admits non-trivial $D$-connected sets. Such a situation is, for example, if $X$ is of the form $V^{q}$ with $\nabla$ being equipped with an inner product and $D$ is a $G$-decomposition of $V^{q}$ for $G$ being the group of all isometries of $V$ acting on $V^{q}$ in the natural way. This may be shown easily by using, for example, the results contained in Topa's paper $\left.{ }^{1}\right)$. We get the same situation if $G$ is a unimodular or conformal group. But if $G=\mathrm{GL}(V)$, then non-trivial $D$-connected sets in $V^{q}$ exist. Incleed, any $D$-admissible set $A \subset V^{q}$ containing the point $0=(0, \ldots, 0) \in V^{q}$ such that $A \neq\{0\}$, is a non-trivial $D$-connected set in $V^{q}$. The main purpose of this paper is to give a method for the construction of other non-trivial $D$-connected sets in $\nabla^{q}$.

Remark 1 . The notion of the $D$-connected set has some connections with the following problem posed to me by Professor Goła:

Let there be given a Lie group $G$ acting on a manifold $X$ and a $G$-invaliant decomposition of $X$ into disjoint submanifolds $X_{1}, \ldots, X_{m}$.

(1) S. Topa, On complete linear, metric, conformal and uninodular classifications of space of all finite sequences of vectors in a given vector space, Zeszyty Naukowo UJ, t. 17 (in press). 
Consider the functional equations

$$
p(T a)=\varphi(x), \quad x \in X, T \in G
$$

and

$$
\varphi_{j}(T x)=\varphi_{j}(n), \quad x \in X_{j}, T \in G(j=1, \ldots, m),
$$

$\varphi: X \rightarrow R, \varphi_{j}: X_{j} \rightarrow R$ being unknowns, and assume tre are giren families $\Phi(j=1, \ldots, m)$ of regular solutions of (2) (for example, the families of all regular solutions). The problem is to determine the family $\Phi$ of all regular solutions $\varphi$ of (1) such that $\varphi \mid X_{j}$ belongs to $\Phi_{j}$ for each $j=1, \ldots, m$.

If for each $j, \Phi_{j}$ contains all constant solutions of (2), then the problem has a positive solution; $\Phi$ contains all constant solutions of (1). From Definition 6 it directly follows that if $X$ is a $D$-connected set with respect; to our $G$-decomposition, then each continuous solution $\varphi$ of ( 1 ) is constant. This means that in our case $\Phi$ is the family of all constant solutions of (1), e.g. the family of all constant functions $\varphi: X \rightarrow R$.

In other cases the problem has not been worked out.

Remark 2. The following fact does not require proof: If $B_{1}$ and $B_{2}$ are concentrating on $A$, then the set $B=B_{1} \cup B_{2}$ also concentrates on $A$. This may be generalized for any family of sets.

Definition 7. A family $\left\{A_{\lambda}\right\}_{\lambda \in A}$ of sets in the space $X$ is said to have the property of finite connectivity iff for each pair $A^{\prime}, A^{\prime \prime}$ of sets in the family there is a finite sequence $A_{1}, \ldots, A_{r}$ of its sets such that (1) $A_{1}=A^{\prime}$, $A_{r}=A^{\prime \prime}$ and (2) $(e=1, \ldots, r)$

$$
A_{\varrho_{0}} \cap \bigcup_{Q \neq \varrho_{0}} A_{Q} \neq \emptyset \quad \text { for all } \varrho_{0} \in\{2, \ldots, r\}
$$

We shall now prove the following:

Proposition 1. Let a family $\left\{A_{\lambda}\right\}_{\lambda \in A}$ of D-comected sets in the space $X$ have the property of finite connectivity and satisfy the condition

$$
\left(\bigcup_{\lambda \in A} A_{\lambda}\right)^{*}=\bigcup_{\lambda \in A} A_{\lambda}^{*}
$$

Then $A=\bigcup_{\lambda \in A} A_{\lambda}$ is a D-conneoted set in $X$.

Proof. From the above condition the first and second properties of $D$-connected sets directly follow. For the third property let us take an arbitlary $D$-function $f$ on $A, D$-continuous on $A^{*}$. Denoting $f_{\lambda}=f \mid A_{\lambda}$ for $\lambda \epsilon \Lambda$ we may state that each function $f_{\lambda}$ is constant. This fact immediately follows from the $D$-connectivity of the set $A$. The constancy of $f$ is implied by the property of finite connectivity of the given family of sets. This completes the proof. 
Remarls 3 . Let us assume that we are given $n$ arbitiary decompositions $D_{1}, \ldots, D_{n}$ of $X$. Then we shall say that we are given an $n$-decomposition of $X$ and denote it by $\left(D_{1}, \ldots, D_{n}\right)$.

A given $n$-decomposition $\left(D_{1}, \ldots, D_{n}\right)$ of $X$ is called quasi-cartesian iff $\inf \left\{D_{1}, \ldots, D_{n}\right\}=X\left({ }^{2}\right)$.

It can be easily seen that: If $\left(D_{1}, \ldots, D_{n}\right)$ is a quasi-cartesian $n$-decomposition of $X$ and $f: X \rightarrow R$ is a $D_{i}$-function for each $i=1, \ldots, n$, then $f$ is necessarily a constant function.

II. $D$-connected sets in $V^{q}$. We have in mind $\mathrm{GL}(V)$-decomposition of $V^{q}$ (see Example 1). We shall use the terminology of GL(T)-orbits instead of $D$-components, GL(V)-admissible sets instead of $D$-admissible sets, and so on. $D$-comnected sets are called $G L(V)$-connected sets. For the construction of certain families of such sets in $V^{r}$ we introduce some denotations and give some lemmas.

Let us write $K=(1, \ldots, q), p=\min (q, \operatorname{dim} V), N$ - the set of all naturai numbers and $X=\nabla^{q}$.

Bу $K(p)$ we mean the family of all subsequences of the sequence $K$ consisting of at most $p$ elements; we also treat the empty sequence, denoted by $\varnothing$, as an element of $K(p)$.

We introduce the mapping $\iota: X \rightarrow K(p)$ (see footnote $\left({ }^{l}\right)$ ), being defined in the following way:

Let $x=\left(x_{k}\right) \in X$. By $n(x)$ we denote the dimension of the subspace $V(x)$ of $V$, generated by the system of vectors $x_{1}, \ldots, x_{q}$, and $\iota(x)$ we define as follows:

1. In the case $x=0$ we put $\iota(x)=\emptyset$;

2. If $x \neq 0$, then we put $\iota(x)=\left(i_{1}, \ldots, i_{n}\right)$, where $n=n(x)$ and $i_{1}, \ldots, i_{n}$ are obtained by use of the following recurrent formula:

$1^{\circ} i_{1}$ is the smallest element in $K$ such that $x_{i_{1}} \neq 0$;

$2^{\circ}$ Suppose that $i_{1}, \ldots, i_{v}$ are determined and that $\nu<n$. Then by $i_{v+1}$ we mean the smallest element in the sequence $i_{v}+1, \ldots, q$ such that the vector $x_{i_{\nu+1}}$ is linearly independent of the system of vectors $x_{i_{1}}, \ldots, x_{i_{v}}$. The mapping $\iota$ is a surjection.

For a given $I \in R(p)$ we denote by $X_{I}$ the coimage of $I$ by the mapping ı. For example, we have $X_{\varnothing}=\{0\}$.

It can be easily seen that each set $X_{I}$ is a $G L(V)$-admissible set. If $I=\emptyset$ or $I=K$ (the case of which may appear only in the situation when $q \leqslant \operatorname{dim} V$ ), then $X_{I}$ is trivial, e.g. consists of one orbit only. If $I \neq \varnothing, \Pi$, then $X_{I}$ is non-trivial. In this case let us denote $J=\Pi \backslash I$ (a subsequence of $K$ ) and introduce the family Mat $(I, J)$ of all matrices

(2) The family of all decompositions of $X$ into disjoint sets forms a lattice partially ordered by refinements. A quasi-cartesian $n$-decomposition $\left(D_{1}, \ldots, D_{n}\right)$ of $X$ is called cartesian iff $\sup \left\{D_{1}, \ldots, D_{n}\right\}=\{X\}$. 
$\left\|a_{j}^{i}\right\|, i \in I, j \in J$, such that the condition

is valid.

$$
j<i \Rightarrow a_{j}^{i}=0
$$

Furthermore, let $n=|I|$, and by $E_{n}$ let us denote the family of all sequences $\left(e_{1}, \ldots, e_{n}\right)$ of linearly independent vectors of the space $V$.

Let $I=\left(i_{1}, \ldots, i_{n}\right), J=\left(j_{1}, \ldots, j_{m}\right)$. For a given $\underset{0}{x \in X}, \underset{0}{x}=\left(\underset{0}{x_{i}}, x_{j}\right)$, $i \in I, j \in J$, the $\mathrm{GL}(\nabla)$-orbit $O(\underset{0}{x})$ of $\underset{0}{x}$ is given by the equations

$$
\begin{aligned}
& x_{i_{\nu}}=e_{v}, \\
& x_{j_{\mu}}=a_{j_{\mu}}^{i_{\nu}} e_{\nu}, \quad\left(e_{v}\right) \in E_{n},
\end{aligned}
$$

where $a_{0}^{i_{j}} i_{p}$ are given by the decompositions

$$
{ }_{0}^{x_{j_{\mu}}}=a_{f_{\mu}}^{i_{y}} x_{i_{p}}
$$

Furthermore, the set $S(\underset{0}{0})$ given by the equations

$$
\begin{aligned}
& x_{i_{\nu}}=x_{0}^{i_{\nu}}, \\
& x_{\rho_{\mu}}=a_{j_{\mu_{0}}}^{i_{\nu}} x_{i_{p}},
\end{aligned} \quad\left\|a_{j_{\mu}}^{i_{\nu}}\right\| \in \operatorname{Mat}(I, J),
$$

is a section (passing through $x$ ) of the space of GL( $\nabla$ )-orbits in $X$ (see footnote $\left({ }^{1}\right)$ ).

We shall prove the following

LEMIA 1. Let $I \in K(p), I \neq K$, and denote $B=X_{I}$. Then a given $\mathrm{GL}(V)$-admissible set $A \subset X\left(X=V^{q}\right)$ is concentrating on the set $B$ iff the condition

$$
\iota(x) \supset I \quad \text { for each } x \in A
$$

\section{is satisfied.}

Proof. For sufficiency let us take an arbitrary $\bar{x} \in A$. We have to show that the GL $(\bar{V})$-orbit $O(\bar{x})$ of $\bar{x}$ reaches the set $B=\bar{X}_{I}$. By assumption we have $\bar{I} \supset I$, wher $\bar{I}=\imath(\bar{x})$. The case $\bar{I}=I$ is trivial. Let $\hat{I} \neq \varnothing$, where by definition $\hat{I}=\bar{I} \backslash I$. Denoting $\bar{J}=K \backslash \bar{I}$ we get for $K$ the decomposition $K=I \cup \hat{I} \cup \bar{J}$. It can be rerified that the sequence $\underset{n}{\vec{x}}=\left(\bar{x}_{n}\right)$ $=\left(\bar{x}_{i}, \bar{x}_{n}, \bar{x}_{n}\right) \in \nabla^{q}$ given by the formulas

$$
\begin{array}{ll}
\bar{x}_{i}=\bar{x}_{i}, & i \in I, \\
\bar{x}_{\hat{i}}=\frac{1}{n} \bar{x}_{\hat{i}}, & \hat{i} \in I, \\
\bar{x}_{n}=\bar{a}_{\bar{j}}^{i} \bar{x}_{i}+\bar{a}_{\bar{j}}^{\hat{i}}\left(\frac{1}{n} \bar{x}_{\hat{i}}\right), & \bar{j} \in J,
\end{array}
$$


where $\bar{a}_{\bar{j}}^{i}$ and $\bar{a}_{\bar{j}}^{\hat{i}}$ are given by the equalities $\bar{x}_{\bar{j}}=\bar{a}_{\bar{j}}^{\bar{i}} \bar{x}_{i}+\bar{a}_{\bar{j}}^{\hat{i}} \bar{x}_{i}$, has the properties that $\bar{x}_{n} \in O(\bar{x})$ and $\lim _{n \mid \infty} \vec{x}$ exists and belongs to the set, $B$. This means nothing but the fact that the orbit $O(\bar{x})$ reaches the set $B$.

To prove the necessity let us assume that the GL(V)-admissible set $A \subset \nabla^{\alpha}$ is concentrating on the set $X_{I}$. We have to show that for each $\bar{x} \in A$ we get $\vec{I} \supset I$, where $\bar{I}=\iota(\bar{x})$. On the contrary, let us suppose that there exists a point $\bar{x}_{0} \in A$ such that ${ }_{0} \neq I$, where $\underset{0}{I}=\iota(\vec{x})$. Then Wo can show that the orbit $O(\bar{x})$ does not reach the set $X_{I}$ and this will lead us to a contradiction of the assumption. Indeed, by the supposition that $\bar{I}_{0} \neq I$ we obtain that $I \cap \bar{J}_{0} \neq 0$. Let us denote $k_{0}=\inf \left(I \cap \bar{J}_{0}\right)$ and distinguish the two cases: (a) $k_{0}=1$, (b) $k_{0}>1$.

In case (a) we obtain the fact that

$$
x_{1} \neq 0 \text { for each } x=\left(\infty_{1}, \ldots, x_{q}\right) \in \bar{X}_{I}
$$

which follows from the fact that $k_{0} \in I$. Simultanously, we have that $k_{0} \in \bar{J}$, which implies that

$$
\bar{x}_{1}=0 \quad \text { for } \operatorname{each} \bar{x}=\left(\bar{x}_{1}, \ldots, \bar{x}_{q}\right) \in \bar{X}_{\bar{I}} .
$$

If we now assume the existence of a sequence $\underset{n}{\bar{x}}=\left(\underset{n}{\bar{x}_{1}}, \ldots, \underset{n}{\bar{x}_{q}}\right) \in O(\underset{x}{x})$, convergent and having the property $\lim _{n \mid \infty} \bar{x}_{n} \in \bar{X}_{J}$, then by virtue of (8) we obtain $\lim _{n \mid \infty} \vec{x}_{1}=0$, which is a contradiction of (7).

In case (b) we follow the same pattern and obtain, instead of (7) and (8), the following two facts:

(9) $\quad x_{k_{0}}$ is linearly independent of $x_{1}, \ldots, x_{k_{0}-1}$

$$
\text { for each } x=\left(x_{1}, \ldots, x_{k_{0}-1}, x_{k_{0}}, \ldots, x_{q}\right) \in X_{I} \text {, }
$$

(10) $\bar{x}_{k_{0}}$ is linearly dependent on $\bar{x}_{1}, \ldots, \bar{x}_{k_{0}-1}$

$$
\text { for each } \bar{x}=\left(\bar{x}_{1}, \ldots, \bar{x}_{k_{0}-1}, \bar{x}_{k_{0}}, \ldots, \bar{x}_{q}\right) \in \underset{0}{X_{\bar{I}}} \text {. }
$$

By these facts the supposition that the orbit $O(\underset{0}{x})$ reaches the set $X_{I}$ leads us to a contradiction.

Lemma 1 is therefore proved.

We shall now give an illustration of this lemma.

ExAMPLE 1. Let $\operatorname{dim} V=3, q=7$. We get $p=3$ and $K=(1, \ldots, 7)$. Putting $I=(2,5)$, let us form the set $\bar{X}_{I}$. We have

$$
X_{(2,5)}=\left\{\left(0, e_{1}, a_{3}^{1} e_{1}, a_{4}^{1} e_{1}, e_{2}, a_{6}^{1} e_{1}+a_{6}^{2} e_{2}, a_{7}^{1} e_{1}+a_{7}^{2} e_{2}\right): a_{j}^{i} \in R,\left(e_{1}, e_{2}\right) \in E_{2}\right\},
$$

where $E_{2}$ is the family of all linearly independent pairs of vectors in $V$. 
If, for example, we take

$$
A=\left\{\left(0, e_{1}, b_{3}^{1} e_{1}, b_{4}^{1} e_{1}, e_{2}, e_{3}, b_{7}^{1} e_{1}+b_{i}^{2} e_{2}+b_{i}^{3} e_{3}\right): b_{j}^{i} \in R,\left(e_{1}, e_{2}, e_{3}\right) \in Z_{3}\right\},
$$

where $E_{3}$ is the family of all linearly independent triples of rectors in $V$, then it may be seen that the set $A$ concentrutes on the set $B=X_{(2,5)}$. However, for example, the set

$$
C=\left\{\left(e_{1}, e_{2}, c_{3}^{1} e_{1}+c_{3}^{2} e_{2}, e_{3}, 0,0,0\right): c_{j}^{i} \in R,\left(e_{1}, e_{2}, e_{3}\right) \in E_{3}\right\}
$$

is not concentrating on the set $X_{(2,5)}$. Moreover, any orbit in $C$ does not reach the set $X_{(2,5)}$, the fact of which is a consequence of the following:

LEMMA 2. For arbitrary $I, \bar{I} \in \bar{K}(p)$ such that $I \subset \bar{I}$ and $I \neq \bar{I}$, we have the following two facts: 1 . Each orbit in $\bar{X}_{\bar{I}}$ reaches the set $X_{I} .2$. Any orbit in $X_{I}$ does not reach the set $X_{\bar{I}}$

This lemma is a direct consequence of Lemma 1 .

Remark 4 . Any orbit in $X_{I}$ does not reach any other orbit in $X_{I}$.

This fact follows from formula (4).

From Remark 4 and Lemma 2 there immediately follows:

LEMIIA 3. If $I, \bar{I} \in R(p)$ such that $I \subset \bar{I}$ and $I \neq \bar{I}$, then denoting $A=X_{I} \cup X_{\bar{I}}$ we get $1^{\circ} A^{*}=X_{I}, 2^{\circ} A$ is concentrating on $A^{*}$.

Now we pass on to the very important

LEMLMA 4. Assume that $I, \bar{I} \in \mathbb{R}(p)$ and $I \subset \bar{I}, I \neq \Pi, \vec{I} \neq \Pi$. Then we can prove the following two facts:

(a) For each $x=\left(x_{i}, x_{j}\right) \in \bar{X}_{I}$ there exists $\bar{x}=\left(\bar{x}_{\bar{i}}, \bar{x}_{\bar{j}}\right) \in \bar{X}_{\bar{I}}$ such that

$$
\bar{x}_{k}=x_{k} \quad \text { for } k \in I \cup \bar{J} ;
$$

(b) For a given $x \in \bar{X}_{I}$ and $\bar{x} \in X_{\bar{I}}$ satisfying condition (11), and for any $\mathrm{GL}(V)$-function $f$ on $B=X_{I} \cup \bar{X}_{\bar{I}}$ being $\mathrm{GL}(V)$-continuous on $X_{I}$ we have

$$
f(\bar{x})=f(x) .
$$

Proof. For proof of (a) let us take an arbitrary $x \in X$ and introduce a point of the form $\left(x_{i}, v_{\hat{i}}, x_{\bar{j}}\right)$ denoted by $\bar{x}$, where $v_{\hat{i}} \in V$ are fixed arbitrarily, provided that $\left(x_{i}, v_{i}\right)$ is a linearly independent system of vectors and, as already denoted, $\hat{I}=\bar{I} \backslash I$. It is evident that the point $\bar{x}$ belongs to $\bar{X}_{\bar{I}}$ and together with the point $x$ satisfies condition (11).

For proof of (b) let us fix $x \in \bar{X}_{I}$ and $\bar{x} \in \bar{X}_{\bar{I}}$ according to condition (11). We consider the sequence $\left.\underset{n}{\bar{x}}=\underset{n}{\left(\bar{x}_{i}\right.}, \bar{x}_{n}, \underset{n}{\bar{x}_{\bar{j}}}\right)$ given by the formulas

$$
\begin{array}{ll}
\bar{x}_{i}=\bar{x}_{i} & \text { for } i \in I, \\
\bar{x}_{\hat{i}}=\frac{1}{n} \bar{x}_{\hat{i}}+x_{i} & \text { for } \hat{i} \in \hat{I}, \\
\bar{x}_{j}=\bar{x}_{\bar{j}} & \text { for } \bar{j} \in \bar{J} .
\end{array}
$$


We easily observe that $1^{0} \bar{x}_{n} O(\bar{x})$ for $n \in N$ and $2^{\circ} \lim _{n \mid \infty} \bar{x}=x$. From the assumed $\mathrm{GL}(V)$-contimuity of $f$ on $X_{I}$ and by equality $2^{\circ}$ we obtain that

$$
\lim _{n \mid \infty} f(\vec{x})=f(x)
$$

and from the constancy of $f$ on each GL( $\nabla)$-orbit (we have assumed that $f$ is a $\mathrm{GL}(V)$-function) we find that

$$
f(\underset{n}{\bar{x}})=f(\bar{x}) .
$$

Equalities (14) and (15) imply (12). The lemma is proved.

For the formulation of fundamental results we introduce the following additional notions:

Defintion 8. A given family $K_{\Omega}=\left\{I_{\omega}\right\}_{\omega \in \Omega}$ of subsequences $I_{\omega}$ of the sequence $K$ is called a covering of $K$ iff $\bigcup_{\omega \in \Omega} I_{\omega}=K$.

By $K_{\Omega}(p)$ wo denote families of subsequences such that $I_{\omega} \in K(p)$ for each $\omega \in \Omega$.

Definrtion 9. We say that a given family $\Pi_{\Omega}=\left\{I_{w}\right\}_{\text {we } \Omega}$ of subsequences of $K$ is olosed from below (or simply olosed) iff for $I_{\Omega}=\bigcap_{\omega \in \Omega} I_{\omega}$ we get $I_{\Omega} \neq \varnothing$ and $I_{\Omega} \in K_{\Omega}$. We call $I_{\Omega}$ the lower bound of $\Pi_{\Omega}$.

For a given closed covering $K_{\Omega}(p)$ of $K$ let us form the sets

$$
X_{\Omega}=\left\{x \in V^{q}: \iota(x) \in K_{\Omega}(p)\right\}
$$

and

$$
X_{I_{\Omega}}=\left\{x \in \nabla^{q}: \iota(x)=I_{\Omega}\right\} \text {. }
$$

Now we may give the first fundamental

THEOREM 1. If $K_{\Omega}(p)$ is a closed covering of $K$ suoh that $|\Omega|>1$, then the set $X_{\Omega}$, defined in (16), is a GL(V)-connected set in $V^{q}$.

Proof. From Lemma 2 and Lemma 3 it immediately follows that if we put $A=X_{\Omega}$, then $A^{*}=X_{I_{\Omega}}, X_{I_{\Omega}}$ having been defined in (17). Next, from Lemma 3 and Remarle 2 we easily obtain that the set $A$ is concentrating on the set $A^{*}$. Thus we see that for our set $A$ the second condition in Definition 6, of $D$-connectivity (in our case $D$ is determined by the group GL $(V)$ ), is valid. To show that also the third condition is valid we distinguish the following three cases:

$1^{0} I_{\Omega}=\varnothing, 2^{\circ} I_{\Omega}=\Pi, 3^{\circ} I_{\Omega} \neq \varnothing, K$.

Case $1^{\circ}$. This case is trivial.

Cas e $2^{\circ}$. In this case the covering $\pi_{\Omega}(p)$ of $K$ is trivial (it consists only of one element, equal to the sequence $K$ ) and the assumption $|\Omega|>1$ in our theorem is not satisfied. 
Case $3^{\circ}$. At first we show that each GL(F)-function $f$ on $A, G L(\nabla)$ continuous on $A^{*}$, is constant on $A^{*}$. For this purpose let us talre an artbitrary point $x_{0} \in X_{I_{\Omega}}$ and consider the section $S(x)$ of the space of $G L(V)$ orbits in $X_{1_{\Omega}}$, given by formulas (5), where $I$ is replaced by $I_{\Omega}$. By the assumption that $f$ is constant on each $\mathrm{GL}(V)$-orbit in $X_{\Omega}$ it is sufficient to show that $f$ is constant on $S(\underset{0}{x})$. To show this we shall make use of Remark 3 and Lemma 4 .

Let us make the denotations

$$
J_{\Omega}=K \backslash I_{\Omega}, \quad I_{\Omega}=\left(i_{1}, \ldots, i_{n}\right), \quad J_{\Omega}=\left(j_{1}, \ldots, j_{n}\right)
$$

and

$$
a_{j_{\mu}}=\left\|a_{j_{\mu}}^{i}\right\| \quad\left(i \in I_{\Omega}\right), \quad j_{\mu} \in J_{\Omega}
$$

and define the function

$$
F\left(a_{j_{1}}, \ldots, a_{J_{m}}\right)=f\left(x_{0}, \ldots, x_{i_{n}}, a_{j_{1}}^{i} x_{i}, \ldots, a_{j_{m_{0}}}^{i} x_{i}\right),
$$

(e.g. $F$ is the restriction of $f$ to $S(\underset{0}{x})$ ). It remains to show that $F$ is constant.

For this purpose, denoting by $U$ the domain of the function $F$ (renember that $\left\|a_{j}^{i}\right\| \in \operatorname{Mat}\left(I_{\Omega}, J_{\Omega}\right)$, see (3)), we shall define a quasi-cartesian $s$-decomposition of $U$, where $s$ is equal to $|\Omega|-1$. If in $\Pi_{\Omega}(p)$ wo fix an arbitraly element $\bar{I}$ different from $I_{\Omega}$, then we may associate with it the following equivalence relation in $\bar{U}$, denoted by $R_{\bar{I}}\left(I_{\Omega} \subset \bar{I}\right.$ and $I_{\Omega} \neq \bar{I}$ imply that $J_{\Omega} \supset \bar{J}$ and $J_{\Omega} \neq \bar{J}$ ),

$$
\left(a_{j_{1}}^{\prime}, \ldots, a_{j_{m}}^{\prime}\right) R_{\bar{I}}\left(a_{j_{1}}^{\prime \prime}, \ldots, a_{j_{m}}^{\prime \prime}\right) \Leftrightarrow a_{\bar{j}}^{\prime}=a_{\bar{j}}^{\prime \prime} \quad \text { for } \bar{j} \in \bar{J} .
$$

Let us denote by $D_{\bar{I}}$ the obtained decomposition of $U$. We shall show that the function $F$ is constant on each element from $D_{\bar{I}}$, e.g. that $F$ is a $D_{\bar{I}}$-function.

We consider the set $X_{\bar{I}}$ and take an arbitrary point $\underset{0}{\bar{x}} \bar{X}_{\bar{I}}$ of the form

$$
\begin{array}{ll}
\bar{x}_{i}=x_{i} & \text { for } i \in I_{\Omega}, \\
\bar{x}_{\hat{i}}=v_{\hat{i}} & \text { for } \hat{i} \in \hat{I}_{\Omega}, \hat{I}_{\Omega}=\bar{I} \backslash I_{\Omega}, \\
\bar{x}_{j}=a_{0}^{i} x_{i} & \text { for } \bar{j} \in \bar{J}\left(i \in I_{\Omega}\right),
\end{array}
$$

where $\underset{0}{x}$ is the point previously fixed in $X_{I_{\Omega}}$ and $v_{\hat{i}}$ and $a_{0}$ are arbitrarily chosen in $Y$ and $R$, respectively, provided that $\left(x_{i}, v_{i}\right)$ form a linearly independent system of vectors. At the same time let us consider the 
family of points $x \in X_{I_{\Omega}}$ given by the formulas

$$
\begin{array}{ll}
x_{i}=x_{0} & \text { for } i \in I_{\Omega}, \\
w_{\hat{i}}=a_{\hat{i}_{0}}^{i} x_{i} & \text { for } \hat{i} \in \hat{I}_{\Omega}\left(i \in I_{\Omega}\right), \\
x_{\bar{j}}=a_{0}^{i} x_{0} x_{i} & \text { for } \bar{j} \in \bar{J}\left(i \in I_{\Omega}\right),
\end{array}
$$

where $a_{i}^{i}$ are the parameters of the family.

It may be seen that for each system of $a_{\hat{i}}^{i}$ the point $x$ given by (21) together with the point $\bar{x}$ satisfies condition (11), where $I$ is replaced by $I_{\Omega}$. Furthermore, for our function $f$ all the assumptions in the second part of Lemma 4 are fulfilled, and by (12) we get the equality $f(\underset{0}{x})=f(x)$. This equality means that for the function $F$ defined in (18) we have the equalities

$$
F\left(a_{i}, a_{0}\right)=F\left(a_{0}, a_{0}\right) \text { for each } a_{\hat{i}}=\left\|a_{\hat{i}}^{i}\right\| \text {. }
$$

Thus we have proved that $F$ is constant on the element from $D_{\bar{I}}$ passing through the point $\left(a_{0}, a_{0}\right) \in U$. But $a_{0} a_{j}=\left\|a_{0}{ }_{j}^{i}\right\|$ appearing in formulas (20) may change arbitrarily, so the function $F$ is a $D_{\bar{I}^{-} \text {function. }}$

If $\bar{I} \neq I_{\Omega}$ varies in $\Pi_{\Omega}(p)$ arbitrarily, then we obtain a family $\left\{D_{\bar{I}}\right\}$ of $s=|\Omega|-1$ decompositions of $U$, being a quasi-catesian $s$-decomposition of $U$. Using Lemma 1 , where $X$ is replaced by $U$ and $f$ by $F$, we obtain that $F$ is a constant function, which was to be proved.

Theorem 1 is therefore proved.

We illustrate this theorem in

ExAMPLe 2. We consider the situation given in Example 1. For the sequence $\mathbb{K}=(1, \ldots, 7)$ we take the following family of its subsequences: $\{(2,3),(1,4),(3,5,7),(6)\}$. This family is a covering for $\mathbb{K}$, but it is not closed. However, a covering of the form $\{(2,3),(1,4),(3,5,7),(6), \varnothing\}$ is a closed covering for $\pi$.

Now we shall give a generalization of Theorem 1. For this purpose we formulate the following two lemmas:

LEMara 5. Let there be given a covering $K_{\Omega}(p) \subset K(p)$ of $K$ and it's olosed subcoverings $\Pi_{\Omega_{e}}(p) \subset K_{\Omega}(p), \varrho=1, \ldots, r$, such that

$$
K_{\Omega}(p)=\bigcup_{Q=1, \ldots, r} K_{\Omega_{e}}(p) \text {. }
$$

Denoting by $I_{\Omega_{e}}$ the lower bound of $K_{\Omega_{e}}(p)$, and by $X_{\Omega_{Q}}$ and $X_{I_{\Omega_{Q}}}$ the sets corresponding, by (16) and (17) respectively, to the covering $K_{\Omega_{e}}(p)$, we may state that

$$
\left(\bigcup_{e} X_{\Omega_{Q}}\right)^{*}=\bigcup_{e} X_{\Omega_{Q}}^{*} \Leftrightarrow \mathrm{V}_{I \epsilon K_{\Omega}(p)} \mathrm{V}_{Q}: I \neq I_{\Omega_{Q}} \Rightarrow I \cap I_{\Omega_{Q}} \neq I
$$


Proof. From Lemma 3 and Remaik 2 we obtain that $X_{\Omega_{0}}^{*}=X_{I_{\Omega_{e}}}$ for each $\varrho$. From Lemma 2 and Remark 4 it follows that the GL(V)-orbit in $X_{I_{\Omega}}$ reaches the $G L(V)$-orbit passing through a given point $\underset{0}{x \in X_{\Omega}}$ iff $I_{0} \neq I_{\Omega_{e}}$ and $I_{0} \cap I_{\Omega_{0}}=I_{0}$, where $I_{0}=\imath(a)$. Furthermore, from Remark 1 we get

$$
\left(\bigcup_{e} X_{\Omega_{0}}\right)^{*} \subset \bigcup_{a} X_{\Omega_{0}}^{*}
$$

All these facts, combined with the definition of the stal operation, complete the proof.

By the same assumptions and denotations as in Lemma 5 we form ulate

LENIIA 6. For each $\varrho_{0} \in\{2, \ldots, r\}$ we have

$$
X_{\Omega_{e_{0}}} \cap\left(\bigcup_{e<e_{0}} X_{\Omega_{e}}\right) \neq \emptyset \Leftrightarrow \mathbb{K}_{\Omega_{Q_{0}}} \cap\left(\bigcup_{e<\Omega_{0}} K_{\Omega_{e}}\right) \neq \emptyset \text {. }
$$

Proof. The given equivalence follows directly from the definition of sets $X_{\Omega_{0}}(\sec (16))$.

By the same denotations as in Lemma 5 we now formulate

TIns0REII 2. Let a covering $\Pi_{\Omega}(p)$ of $K$ be given such that there exists a sequence of closed subcoverings $K_{\Omega_{0}}(p), \varrho=1, \ldots, r$, satisfying the following conditions :

$$
\begin{aligned}
& 1^{0} K_{\Omega}(p)=\bigcup_{e} K_{\Omega_{Q}}(p), \\
& 2^{\circ} \nabla_{I e K_{\Omega}(p)} \nabla_{Q}: I \neq I_{\Omega_{e}} \Rightarrow I \cap I_{\Omega_{Q}} \neq I, \\
& 3^{0} K_{\Omega_{e_{0}}} \cap\left(\bigcup_{Q<e_{0}} K_{\Omega_{e}}\right) \neq \emptyset \text { for eaoh } \varrho_{0} \in\{2, \ldots, r\} .
\end{aligned}
$$

Then the set $X_{\Omega}$ given for our covering $K_{\Omega}(p)$ by (16) is a $\mathrm{GL}(\mathrm{T})$-connected set.

Proof. By wirtue of Theorem 1 we obtain for each $\varrho$ that the set $X_{\Omega}$ is a $\mathrm{GL}(V)$-connected set. By assumptions $2^{\circ}$ and $3^{\circ}$ and Lemmas 5 and 6, respectively, we may state that Proposition 1 can be applied to the family $\left\{X_{\Omega_{e}}\right\}$ of sels in $X=\nabla^{q}$. In consequence, we obtain that $X_{\Omega}$ really is a GL $(V)$-connected set, which was to be proved.

ExAIPLE 3. In the case of Example 1 the covering $\{(1,2,3),(2,3),(3,4,5),(2,3,6),(3,5,7),(2,5,7),(3,6),(3),(2)\}$ of the sequence $\mathbb{K}=(1, \ldots, 7)$ satisfies all the assumptions of Theorem 2 . Indeed, if we put $K_{\Omega_{1}}(3)=\{(1,2,3),(3,4,5),(3,5,7),(3,6),(3)\}$ and $K_{\Omega_{2}}(3)=\{(1,2,3),(2,3),(2,3,6),(2,5,7),(2)\}$, then we obtain a twoelement sequence of subcoverings which are closed and satisfy conditions $1^{0}-3^{0}$. 